\title{
Application of Digital Minimally Invasive Medicine and Visual Simulation in Surgery
}

\author{
Xiaolu Zhang ${ }^{1,2, a^{*}}$, Meihong Du ${ }^{1,2, b}$ \\ Jiangang Zhang ${ }^{1,2, c}$, and Hongjie $\mathrm{Li}^{1,2, \mathrm{~d}}$ \\ ${ }^{1}$ Henan Province Hospital of Traditional Chinese Medicine, 450002.Zhengzhou, 450000, Henan, P. \\ R. China \\ ${ }^{2}$ The Second Affiliated Hospital of Henan University of Traditional Chinese Medicine, 450000, \\ Henan, P. R. China \\ azhangxiaoluhntcm@163.com, bdumeihonghntcm@163.com, \\ 'zhangjianganghntcm@163.com, ${ }^{\mathrm{d}}$ lihongjiehntcm@163.com
}

Keywords: Digital medicine; Simulation surgery; Image segmentation; 3D reconstruction; Surgery

Abstract. Surgery into the new minimally invasive surgery era, minimally invasive surgery more dependent on all kinds of advanced technology and equipment applications, and the traditional operating room in the design is difficult to meet the minimally invasive surgery to carry out, so the development of minimally invasive surgery the operating room is particularly important. Combined with the hospital minimally invasive operating room design examples, proposed a new era of minimally invasive surgery need to pay attention to the issue, put forward the development and construction of digital surgery application advantages and future development. Three - dimensional reconstruction technique based on helical CT abdominal organ scan data and clinical application of visual simulation in surgery. Visual simulation of Free Form Modeling System and PHANTOM software. The three-dimensional reconstruction technique and visual simulation operation of digital medicine can be applied to the clinical practice, and it has great application value in the aspects of individualized surgical procedure, risk assessment, clinical teaching and surgical training.

Traditional surgical surgery has matured, limiting the development of surgery is not a surgical technique itself, but around the surgical software ${ }^{[1]}$. With the development of digital medicine and minimally invasive surgery, digital minimally invasive technology came into being, refers to the three-dimensional reconstruction, visual simulation surgery and other digital medical technology applied to surgical clinical work, to achieve surgical minimally invasive ${ }^{[2]}$. Since the 20th century, 90 years, a combination of computer image processing and analysis, realistic computer graphics, virtual reality and other technical image of the three-dimensional visualization technology has been a hot research and application at home and abroad, in the past 10 years The rapid development ${ }^{[3]}$. In the preoperative disease diagnosis, surgical design and teaching and simulation of surgical training and other aspects of digital medical technology is an important area of application.

Significant development of minimally invasive surgery by the surgeon, patients and clinical medical engineering staff unanimously approved, minimally invasive treatment technology to effectively reduce the organization's surgical damage, more conducive to the recovery of the body function advantages, leading to modern hospital surgery minimally invasive technology applications Continue to develop in depth, such as endoscopy, endoscopic surgery, interventional therapy, directed guidance surgery, telemedicine, and even microsurgery and gene therapy. In order to adapt to the development of minimally invasive treatment technology, we need to develop a new era of minimally invasive operating room, the design of a new digital minimally invasive operating room. The operating room as an independent space for surgical treatment of the surgeon has been around how to develop safer and more effective surgery. The traditional operating room follows the following basic rules: adequate space to accommodate the surgeon and various life monitoring support equipment and other facilities to optimize the operation (such as better surgical lighting, clean air, etc. At the same time, with the development of minimally invasive surgery, there have been many new technologies and equipment, modern digital integrated operating room design 
principle also added some new content, mainly: (1) reasonable space layout, centralized and effective management of all Equipment; (2) the ergonomic design principles that meet the operation of the surgeon; (3) the design requirements for digital information integration; and (4) adapt to the future design of advanced technology ${ }^{[4]}$.

\section{Reasonable Space Layout}

Centralized and effective management of all equipment minimally invasive operating room is a significant feature of all kinds of digital equipment and surgical equipment to become its standard configuration, because so many indoor equipment into an operating room, such as equipment between the power supply, cable As well as various types of pipes and so make the operating room becomes chaotic, affecting the movement of the operator, and is not conducive to maintaining the operating room clean state ${ }^{[5]}$. Therefore, centralized and effective management of all the equipment to facilitate the flow of surgical personnel and items become a modern minimally invasive operating room design of one of the most important principles. The application of computer centralized control technology can effectively solve the defects of traditional design, design and development of intelligent centralized control and management system will be all kinds of equipment to achieve network connectivity, through the touch screen or RF remote control of the corresponding equipment to the necessary operation and control. This will not only reduce the frequency of leaving the sterile area during the operation of the medical staff, but also the effective control of the equipment (such as the mirror equipment, monitor the screen image, indoor air and surgical lighting adjustment, etc.), but also in the operating table On the basis of the basic status of patients can be recorded in real time, including physical care, anesthesia depth, surgical process images and other document information. Efficient centralized equipment management can save manpower, improve the efficiency of the surgeon and reduce the unnecessary operation of the nurses to move, change the minimally invasive operation of many indoor equipment disorderly situation, while the equipment is conducive to safe use, control of potential bad Events and the occurrence of human medical malpractice.

\section{Ergonomic Design Principles for the Operation of the Surgeon}

Minimally invasive surgery compared to traditional open surgery, the need for more advanced technology and equipment to master the difficulty of surgery itself is also greatly increased, the medical staff, especially the surgeon of the operating process requires more refined, any uncomfortable position or Posture will affect the doctor's operation ${ }^{[6]}$. Therefore, in the modern minimally invasive operating room in accordance with the ergonomic principles of each subtle design can not be ignored. Most minimally invasive surgery is through a monitor or microscope to observe the surgical field of vision, and in this visual operation greatly affected the surgeon's three-dimensional sensory and tactile feedback, increased hand-eye coordination difficulty. Therefore, in the design, surgical vision monitor should be able to flexibly adjust to meet the requirements of different surgeons, in conditions permitting, you can ensure that you can install multiple video monitors in the operating room, and can allow doctors to adjust their own ; Surgical bed can be adjusted according to minimally invasive surgery to meet the doctor's best position to surgery to reduce the doctor's physical fatigue and muscle soreness; hanging tower design allows more equipment to leave the ground to facilitate the operation of doctors and nurses; At the same time, the operating room floor should be clean, to avoid delays in a variety of pipeline and cause potential danger. The design principle of ergonomics is to coordinate the interaction between the three elements of good people, machine and environment, so that the three elements can get the most efficient, healthy, safe and comfortable. Modern minimally invasive techniques are increasingly applied to the field of surgery, according to the principle of ergonomic rational design of minimally invasive surgical instruments and equipment, in addition to its function, the equipment and equipment when the operation of the comfort, safe and reliable, environmental factors (such as lighting Etc.) is also the primary consideration ${ }^{[7]}$. After a comprehensive analysis and design will be 
conducive to the health of medical personnel, while patients will also be more safe treatment, and promote the efficiency of the entire operating room and efficiency.

\section{Digital Information Integration Design Requirements}

Many domestic and foreign teaching hospitals have been committed to the development of the operating room digital information, and has made great progress, such as operating room teaching and broadcasting system has been able to achieve audio and video two-way multi-channel transmission and interaction, some areas of the hospital PACS Extended to the operating room for the surgeon during the operation to watch. With the development of minimally invasive surgery, the operating room also requires more minimally invasive surgery aids such as surgical ultrasound equipment, endoscopic imaging systems, digital image acquisition systems and other inspection devices, which provide surgery Accurate and timely information, but also increased the difficulty of managing a large number of information ${ }^{[8] . ~ T h e r e f o r e, ~ t h e ~ m o d e r n ~ m i n i m a l l y ~ i n v a s i v e ~ o p e r a t i n g ~}$ rooms in addition to the digital information design, more importantly, to achieve the rapid changes in digital information integrated management control. At present, the intelligent level of computer technology has been able to centrally manage this digital information, and all the information for effective screening to filter out the normal information, so that the surgical staff to determine and solve the need to deal with the sudden and dangerous signals, the work of the pressure to improve efficiency.

\section{Adapt to the Future of Advanced Technology to Expand the Design Concept}

The technology of digital information age is very fast, three-dimensional visual reconstruction technology, virtual reality technology and surgical robot technology will replace the existing minimally invasive surgical model, as the future standardization of surgical methods. Medical engineering experts often in the new technology appears the first time the new technology has been applied to clinical design, it can be said that surgery also entered a digital computer era. In the design of modern digital minimally invasive operating room, we need to foresee the future development and application of new technologies to maintain a certain degree of scalability, so that the operating room in the future because of difficult to upgrade and had to completely rebuild and spend more money. At the same time, the standard professional minimally invasive operating room not only to meet the needs of ordinary surgery, but also can be applied to other surgical specialist, obstetrics and gynecology and orthopedics. The operating room device can be differentiated by specialties, but should be able to meet the needs of all professional surgeons using advanced new technology to efficiently perform all kinds of complex minimally invasive surgery. Surgery in minimally invasive surgery relies on high-end advanced technology and computer program applications; the operating room is designed to allow the operation of the operation of advanced surgical equipment system to maximize the effectiveness of patients to provide the best service.

Minimally invasive surgery and traditional surgical surgery is fundamentally different, it needs to be familiar with the new technology application and master, increased the difficulty. Young doctors training in the operating room at the beginning of the design to give full consideration, the operating room can be collected panoramic surgery, surgery and endoscopes or microscopes and other high-definition video information, and through the broadband network to the classroom Or academic conference site, the two-way audio and video real-time interaction to communicate. In order to compensate for the lack of on-site practice, some operating room design also set aside the application of virtual reality technology to develop surgical simulation training platform function interface, so that the operating room can be used as a regular training of new students or young doctors ideal place.

\section{Conclusions}

The application of three-dimensional reconstruction and simulation of human computer in medical 
application originated from the visualization of complex three-dimensional medical anatomical data. On the basis of it, a three-dimensional simulation environment was established for surgical and surgical procedure. Minimally invasive surgery has an encouraging application prospects. High-end technology, guided by molecular biology, genetics, imaging, light-guiding fibers, robots, and computer aids, is used in minimally invasive surgery to make the design of minimally invasive operating rooms change. The most popular study abroad is the robot-assisted surgical system, which is considered a new generation of surgical methods in the future, with a high degree of flexibility, three-dimensional visual display more accurate characteristics, may be widely used in the near future clinical. At present, the domestic research in this area is relatively backward, so the early development of independent intellectual property rights and more accurate and accurate computer robotic surgical system, and its application in minimally invasive surgical practice, is China's medical engineering staffs face problems. The advent of minimally invasive surgery to the development of surgical surgery to a new field, and modern operating room design and application of the need to integrate a variety of advanced technology to enable surgeons in a safe and comfortable environment to complete the operation efficiently, Such an operating room will provide us with better medical services and generate more benefits.

\section{References}

[1] Bibas L, Thanassoulis G. Fractional Flow Reserve-Guided Multivessel Angioplasty in Myocardial Infarction. N Engl J Med. 2017. 377(4): 396-7.

[2] Li X, Wang T, Yao L, et al. The safety and effectiveness of robot-assisted versus laparoscopic TME in patients with rectal cancer: A meta-analysis and systematic review. Medicine (Baltimore). 2017. 96(29): e7585.

[3] Shibata E, Takao H, Amemiya S, Ohtomo K. 3D-Printed Visceral Aneurysm Models Based on CT Data for Simulations of Endovascular Embolization: Evaluation of Size and Shape Accuracy. AJR Am J Roentgenol. 2017. 209(2): 243-247.

[4] Miftahussurur M, Syam AF, Nusi IA, et al. Surveillance of Helicobacter pylori Antibiotic Susceptibility in Indonesia: Different Resistance Types among Regions and with Novel Genetic Mutations. PLoS One. 2016. 11(12): e0166199.

[5] Sleightholm R, Wahlmeier S, Carson JS, Drincic A, Lazenby A, Foster JM. Massive adrenal vein aneurysm mimicking an adrenal tumor in a patient with hemophilia A: a case report and review of the literature. J Med Case Rep. 2016. 10(1): 343.

[6] De Moura DT, Chacon DA, Tanigawa R, et al. Pancreatic metastases from ocular malignant melanoma: the use of endoscopic ultrasound-guided fine-needle aspiration to establish a definitive cytologic diagnosis: a case report. J Med Case Rep. 2016. 10(1): 332.

[7] Mohammed N, Subramanian V. Clinical relevance of endoscopic assessment of inflammation in ulcerative colitis: Can endoscopic evaluation predict outcomes. World J Gastroenterol. 2016. 22(42): 9324-9332.

[8] Wang J, Xia SJ, Liu ZH, et al. Inguinal and subinguinal micro-varicocelectomy, the optimal surgical management of varicocele: a meta-analysis. Asian J Androl. 2015. 17(1): 74-80. 